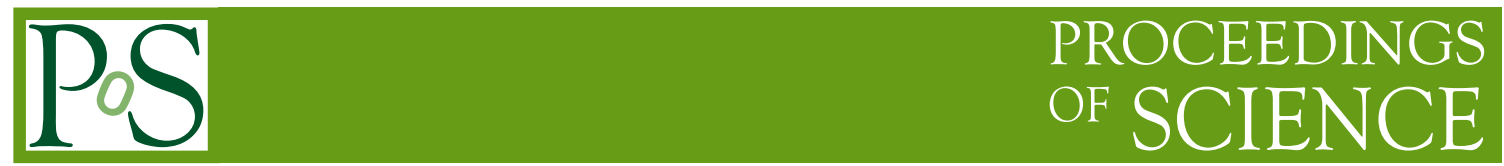

\title{
Level-1 trigger algorithms at CMS for the HL-LHC
}

\author{
Jingyan $\mathrm{Li}^{a, *}$ on behalf of the CMS Collaboration \\ ${ }^{a}$ Northeastern University, \\ 360 Huntington Ave, Boston, United States \\ E-mail: jingyan.li@cern.ch
}

In light of the High-Luminosity LHC (HL-LHC), the Compact Muon Solenoid (CMS) experiment is planning to upgrade its trigger system. The Level-1 (L1) trigger will benefit from the addition of tracking information as well as high granularity information from the upgrades of subdetectors. In this report, we present a panel of algorithms to select events containing electrons, photons, jets, missing transverse energy and hadronically-decaying $\tau$ leptons. These algorithms aim to provide a maximum trigger efficiency while keeping the event rate under control. The performance of these algorithms are studied using simulated samples produced choosing a center of mass energy of $14 \mathrm{TeV}$ and an average of 200 collisions per bunch crossing.

40th International Conference on High Energy physics - ICHEP2020

July 28 - August 6, 2020

Prague, Czech Republic (virtual meeting)

\footnotetext{
${ }^{*}$ Speaker
} 
Planned to start in 2027, the High-Luminosity LHC (HL-LHC) will reach a peak instantaneous luminosity of $7 \times 10^{34} \mathrm{~cm}^{-2} \mathrm{~s}^{-1}$ along with 200 average proton-proton collisions per bunch crossing (pileup). The increased luminosity will open up the opportunities for ambitious physics programs including precision Standard Model measurements and searches for physics beyond the Standard Model. In order to maintain a low trigger threshold even with the harsh environment, the Compact Muon Solenoid (CMS) experiment is planning to replace its entire Level-1 (L1) trigger and data acquisition system [1]. The upgraded L1 trigger is able to run sophisticated algorithms inspired by the ones used in the offline reconstruction. High performance particle-flow (PF) based algorithms have been developed to take advantage of the full detector information that is available at L1 [2]. Simpler algorithms that only take subdetector information have also been developed to add robustness. In the following, we describe the main characteristics of these algorithms and their performance evaluated based on simulated collision data of the HL-LHC.

\section{1. $e / \gamma$ algorithms}

The reconstruction of $e / \gamma$ candidates in the barrel calorimeter will benefit from the upgraded granularity, which is 25 times higher than the current trigger input. With a much better position resolution, the $e / \gamma$ algorithm is able to perform precise track-cluster matching and calorimeter isolation. The entire electromagnetic calorimeter (ECAL) endcap will be replaced with a high granularity calorimeter (HGCAL) which provides detailed shower information. A BDT is implemented to help identify signal candidates formed in the endcap.

L1 tracks are propagated to the calorimeter surface to match with calorimeter clusters. An elliptical cut in the $\eta-\phi$ plane is applied and illustrated in Fig. 1 (left). Fig. 1 (center) demonstrates the small efficiency drop which is largely driven by track reconstruction. Fig. 1 (right) demonstrates the resulting sizable rate reduction.
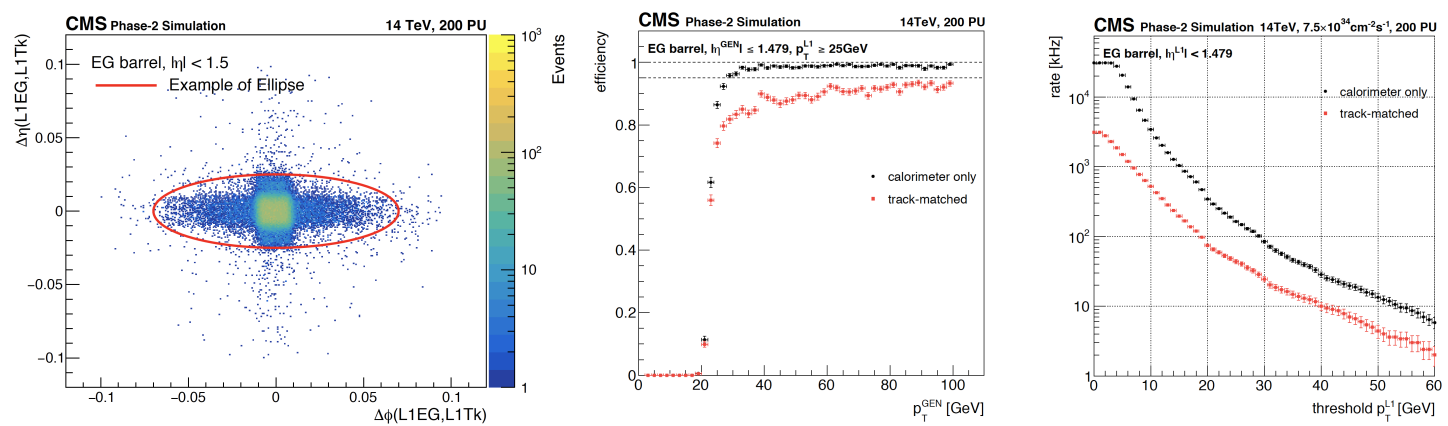

Figure 1: (Left) $\Delta \eta$ vs $\Delta \phi$ distances between calorimeter clusters and the closest L1 track. (Center) single electron efficiency as a function of the generated $p_{\mathrm{T}}$. (Right) trigger rate as a function of the cluster $p_{\mathrm{T}}$.

\section{Jet finding and $E_{\mathrm{T}}^{\mathrm{miss}}$ algorithms}

The calorimeter-only jet finding algorithm is largely inspired from what is currently being used at L1 but exploiting more granular information. This algorithm has a very good efficiency and adds robustness to the system by providing a standalone option. Track-only algorithms have been developed for both jets and energy sums. These algorithms use stringent track purity requirements 
to reduce the number of misidentified tracks, and consequently the trigger threshold.

The PF-based jet finding algorithm uses the Pile Up Per Particle Identification (PUPPI) algorithm to suppress the contribution from pileup [3]. This allows for a larger jet size to maximize the trigger efficiency to be implemented. This algorithm has an improved jet response and energy resolution when compared to calorimeter-only or track-only jet finding. Both the efficiency and the rate produced by this algorithm is close to that of the offline AK4 algorithm when using PUPPI inputs. This algorithm has been implemented using High-Level Synthesis (HLS) and tested in a hardware demonstrator. A comparison of combined matching and turn-on efficiency for various jet finding algorithms is shown in Fig. 2 (left).

A PF-based $E_{\mathrm{T}}^{\text {miss }}$ algorithm that takes PUPPI inputs has been developed and implemented in firmware using HLS. This algorithm has a shaper and lower turn-on curve than the track-only algorithm, which is demonstrated in Fig. 2 (center).
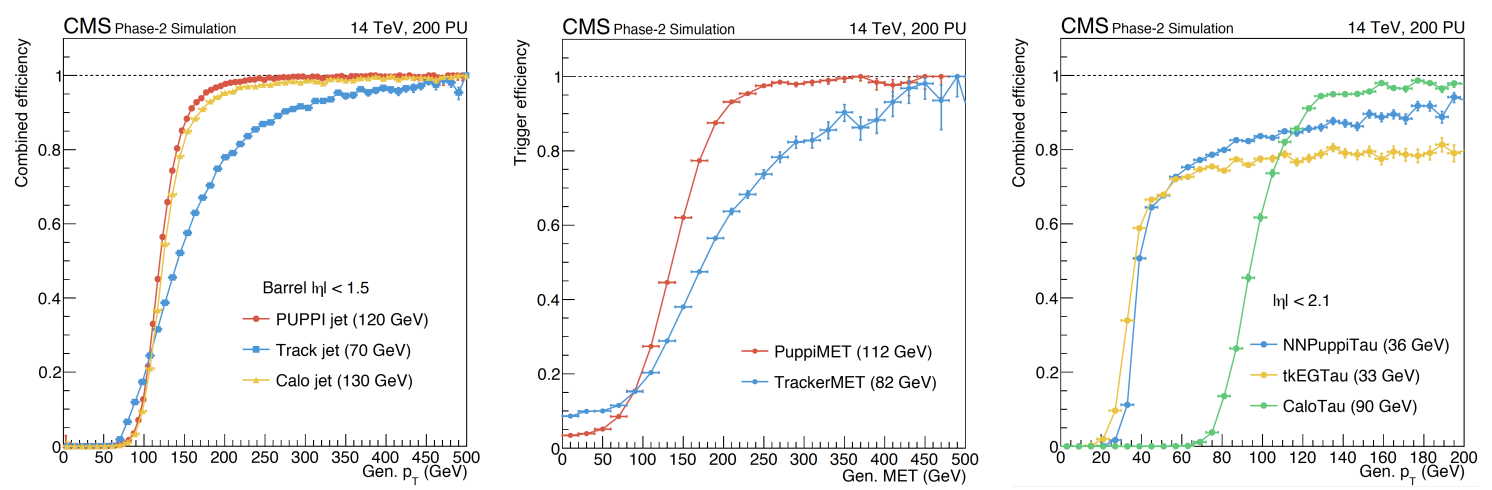

Figure 2: Comparison of combined matching and turn-on efficiency for algorithms with different levels of sophistication, for jet finding (left), $E_{\mathrm{T}}^{\text {miss }}$ (center) and $\tau_{h}$ (right).

\section{3. $\tau_{h}$ algorithms}

To take advantage of the detailed information provided by the HGCAL, the calorimeter-only $\tau$ reconstruction algorithm implements a BDT to optimize object identification in the endcap. The track+e $/ \gamma$ algorithm builds up $\tau$ candidates by associating e/ $\gamma$ clusters to the track-only objects. This algorithm delivers a solid performance that is comparable to more sophisticated algorithms.

The PF-based approach takes either PUPPI or PF candidates and utilizes a neural network to help identify $\tau$ candidates. A cut is placed on the neural network output to reduce the rate for lower- $p_{\mathrm{T}}$ particles. An FPGA based implementation of this algorithm is performed and the use of the FPGA resources is optimized to allow for more $\tau$ candidates.

A comparison of the algorithm performance is shown in Fig. 2 (right).

\section{References}

[1] The Phase-2 Upgrade of the CMS Level-1 Trigger, CMS, CERN-LHCC- 2020-004, CMS-TDR-021.

[2] Particle-flow reconstruction and global event description with the CMS detector, CMS, JINST 12 P10003 (2017).

[3] Pileup Per Particle Identification, D. Bertolini, P. Harris, M. Low, and N. Tran, JHEP 10 (2014) 059. 